\title{
New Energy Aggregators Optimal Dispatching Strategies Based on Price Incentive Agreement
}

\author{
Jie $\mathrm{Yu}^{1 *}$, Xinsen Zhang ${ }^{1}$, Jiaqi Yang ${ }^{1}$, Mengfu Tu${ }^{2}$, Peng $\mathrm{Li}^{3}$ and Qia Ding ${ }^{2}$ \\ ${ }^{1}$ School of Electrical Engineering, Southeast University, Nanjing, Jiangsu, 210096, China \\ ${ }^{2}$ NARI Group Corporation, Nanjing, Jiangsu, 210096, China \\ ${ }^{3}$ Guodian Nanjing Automation Co.,Ltd, Nanjing, Jiangsu, 210096, China
}

\begin{abstract}
The ubiquitous power Internet of things (UPIoT) can realize the wide interconnection of all links of the power system. Based on this background, this paper proposes the price incentive agreement of distributed new energy aggregators and establishes distributed dispatching architecture. Firstly, the information transmission matrix of distributed new energy aggregators with the property of double random matrix is established, and the distributed sub-gradient algorithm based on information transmission matrix is used to solve the distributed dispatching model of new energy aggregators. Finally, the feasibility and effectiveness of the optimization model and its solution method are analyzed through simulation examples, and the information interrupt, information error that may be encountered are also discussed.
\end{abstract}

\section{Introduction}

Various types of distributed resources could be aggregated into new energy aggregators through advanced communication technologies and control strategies. Under the condition that a large number of distributed new energy sources are connected, effective control and management of distributed new energy sources can be realized to obtain logically aggregated power generation[1]. In the context of "building a fullservice ubiquitous power Internet of Things"[2], new energy aggregators' optimal dispatch can make full use of advanced information technology and communication technology to realize interconnection and interaction of distributed new energy aggregators nodes to realize its state perception and control.

The number of distributed new energy resources that need to be coordinated and optimized within the new energy aggregators is huge. The centralized dispatch requires establishing a centralized control center and maintaining real-time communication control between each node and the centralized control center. Therefore, there are limitations in dispatching flexibility and robustness. Under the openness, scalability and other core features of ubiquitous power IoT[3], distributed new energy optimal dispatch is a mode of assigning tasks and workloads among participants, and due to its "decentralization" features, it does not require the construction of a centralized control center and can make full use of the existing information communication architecture of the ubiquitous power IoT. The organizational structure is simple and the construction cost is close to zero. Therefore, this distributed model coincides with the idea of the ubiquitous power IoT. However, the feasibility and convergence of the distributed new energy optimal dispatch is the key to the problem.

Experts and scholars have studied the optimal dispatching operation of distributed new energy supplies. Reference [4] proposed a distributed control method for multi-energy interconnection. On the basis of ensuring the accurate distribution of load power among distributed power sources including new energy, the output voltage phase angle and amplitude of each distributed power source are kept consistent, so that the calculated current between distributed power sources can be reduced or even eliminated; reference [5] proposed a "consistent term plus correction term" dispatching algorithm, the consistent term ensures that the incremental cost converges to the optimal value, and the correction term is based on the actual operation of the physical equipment.The above consistent innovation method makes the setting of the master node unnecessary. Reference [6] analyzed the economic characteristics of distributed generation systems, and proposes a distributed power optimal operation strategy based on cooperative game. Based on the traditional distributed new energy dispatch, environmental factors are considered to better realize the optimal operation dispatch of power system. According to reference [7], for the current situation of a large number of distributed new energy access, a distributed new energy optimal method based on market control is proposed. According to reference [8], considering the uncertainty of the plugand- play state of the microgrid, a distributed interactive

"Corresponding author's e-mail: zhangxinsen123@163.com 
optimal method that can deal with the change of the dimension of the variable is proposed, which converges to the global optimal solution through iteration and information interaction.

In summary, there are quite research results for the optimal dispatch of distributed new energy supplies. However, under the background of the development of ubiquitous power Internet of Things, how to adapt to its information transmission characteristics and consider its possible information transmission problems has not yet been studied furtherly. This paper studies the information transmission link characteristics under the ubiquitous power Internet of Things, and proposes a distributed new energy optimal algorithm with better convergence characteristics. At the same time, considering the influence of information error and delay on the optimization results between the new energy aggregators nodes, it has certain theoretical breakthrough and engineering practicability.

\section{Distributed new energy aggregators optimization dispatching architecture}

Due to the small capacity, large quantity and scattered geographical distribution of distributed new energy sources, it is difficult to establish a unified control center for them. Centralized dispatch of all distributed new energy sources leads to high costs and reduced economic benefits. The distributed dispatching architecture based on the power ubiquitous Internet of Things is an application architecture that distributes tasks and workloads between peer nodes. There is no sub-level between nodes, which has the characteristics of decentralization. Therefore, in the context of the ubiquitous power IoT, this paper establishes a optimization dispatching architecture as shown in Figure. 1 to achieve optimal dispatch of distributed new energy sources.

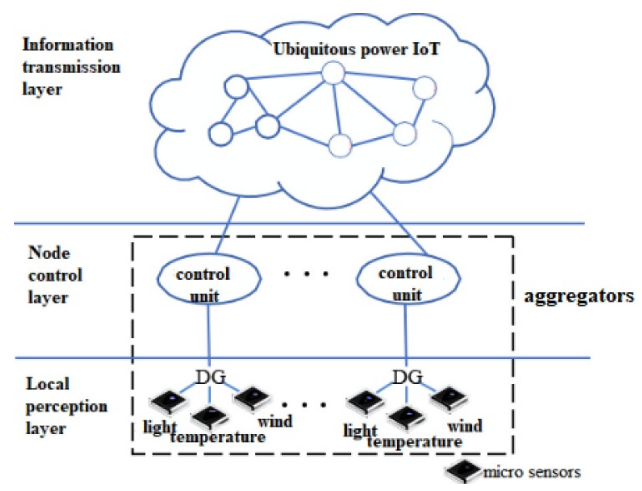

Figure 1. Distributed new energy aggregators optimization dispatching architecture

The dispatching architecture mainly includes three levels. The first layer is the local perception layer, which is composed of a plurality of sensors and is deployed around the distributed new energy sources to realize the ubiquitous perception of the physical state. The second layer is the node control layer, which mainly includes the control decision units of each distributed power source to achieve effective control of each distributed new energy supply. The third layer is the information transmission layer, which realizes the transmission and distribution of distributed new energy running status information through the information transmission network and the traditional physical transmission network.

\section{Distributed new energy aggregators optimization dispatching model}

Distributed power has the characteristics of small scale, uneven distribution and unstable output. Under the technical support of ubiquitous power IoT, the objective of the optimization dispatching model is to achieve the lowest total dispatching cost under the condition of a certain total dispatching task.

\subsection{Price Incentive Agreement}

This paper proposes a price incentive protocol for distributed new energy sources, which enables optimal allocation of power generation and coordination of benefits for distributed new energy sources.

In this paper, under the distributed new energy aggregators optimization dispatching architecture, the aggregator signs the agreement with the distributed new energy sources. This paper designs the price incentive agreement as follows[9]:

$$
c_{i}\left(P_{D G i}\right)=\alpha_{i} P_{D G i}+\beta_{i}
$$

Where, $\alpha_{i}$ represents the incentive coefficient, $\beta_{i}$ reprsents a fixed coefficient. $P_{D G i}$ represents the output control variable of the $i_{\text {th }}$ distributed new energy supply.

Under this price agreement, the dispatching cost of the agent to the distributed new energy source is:

$$
F_{i}=\int_{0}^{P_{D G i}} c_{i}\left(P_{D G i}\right) d P_{D G i}=\frac{\alpha_{i}}{2} P_{D G i}^{2}+\beta_{i} P_{D G i}
$$

\subsection{Objective function}

The agent pays the corresponding dispatching cost by signing a protocol with each distributed new energy source. In this paper, the minimum cost of the distributed new energy source is optimized as the optimization goal, and the following objective function is established:

$$
\min F=\sum_{i=1}^{N} F_{i}=\sum_{i=1}^{N} \frac{\alpha_{i}}{2} P_{D G i}^{2}+\beta_{i} P_{D G i}
$$

Where, $F$ represents the total dispatching cost of all new energy aggregators.

\subsection{Constraints}

Dispatching tasks of all new energy aggregators: 


$$
\sum_{i=1}^{N} P_{D G i}=P_{t o t a l}
$$

Where, $P_{\text {total }}$ represents the total dispatching tasks to be completed.

Output capacity constraints of each distributed new energy source:

$$
0 \leq P_{D G i} \leq P_{D G \operatorname{maxi}}
$$

Where, $P_{D G \max }$ represents the upper limit of the capacity of the distributed power supply.

Wind power output real-time power constraint:

$$
0 \leq P_{D G i} \leq P_{\text {real }-W T, i}
$$

Where, if the distributed new energy supply i represents wind power, the real-time power constraint of wind power output must be satisfied. $P_{\text {real-WT,i }}$ is the realtime available wind power in this state.

Photovoltaic output real-time power constraints:

$$
0 \leq P_{D G i} \leq P_{\text {real }-P V, i}
$$

Where, if the distributed new energy supply $i$ is photovoltaic, the real-time power constraint of the photovoltaic output is satisfied. $P_{\text {real }-P V, i}$ represents the real-time available photovoltaic power in this state.

\section{Distributed optimization algorithm}

In this paper, the distributed sub-gradient algorithm based on information transmission matrix is used to solve the new energy aggregators optimal dispatching model. The main idea is to establish an information transmission matrix, and normalize it into a double random matrix form. The double random matrix is the guarantee of the convergence of distributed sub-gradient algorithm[10]. This paper proposes the definition of effective path of information transmission and the calculation method of information transmission matrix.

\subsection{Information Transmission Matrix}

The information transmission matrix describes the transmission path connection relationship in the information transmission network, and it is also an important basis to design the distributed optimization algorithm. The number of effective paths of information transmission between each distributed new energy sources $R_{i j}$ is the element in the matrix $R_{N \times N}$, which is called an information transmission matrix. The larger the effective paths number, the stronger the information transmission reliability, and the accessibility, timeliness and accuracy of the information flow are higher. Only when the information transmission matrix is transformed into a double random matrix by means of symmetric normalization can it meet the requirements of distributed sub-gradient optimization algorithm.

Take $A_{N \times N}$ as an information transmission matrix with the properties of a double random matrix, and $a_{i j}$ represents each element in the matrix. The overall steps are as follows:

(1) Normalize the first column element of $R_{N \times N}$.

$$
a_{i 1}=R_{i 1} / \sum_{i=1}^{N} R_{i 1}, \quad i=1 \cdots N
$$

(2) Let $a_{1 i}=a_{i l}(i=1, \ldots, n)$ to meet the conditions of a double random matrix.

(3) Normalize the second column element of $R_{N \times N}$ from the second row to the $\mathrm{N}_{\text {th }}$ row, and must satisfy the condition that the column sum is 1 .

$$
a_{i 2}=R_{i 2} / \sum_{i=2}^{N} R_{i 2}\left(1-a_{12}\right), \quad i=2 \cdots N
$$

(4) Let $a_{2 i}=a_{i 2}(i=1, \ldots, n)$ to meet the conditions of a double random matrix.

(N)Normalize the N-1 column element of $R_{N \times N}$ from the $\mathrm{N}-1$ row to the $\mathrm{N}_{\text {th }}$ row, and must satisfy the condition that the column sum is 1 .

$$
(\mathrm{N}+1) \text { Let } a_{N, N}=1-\sum_{m=1}^{N-1} a_{m, N} \text {. }
$$

At this time, matrix $A_{N \times N}$ is an information transmission matrix with double random matrix properties, which evolves from the information transmission matrix, so it can be used to represent the information transmission line connection in the energy network.

\subsection{Distributed optimization algorithm}

The optimization problem to be solved in this paper can be written as follows:

$$
\left\{\begin{array}{l}
\min \sum_{i=1}^{N} F_{i} \\
\text { s.t. } \sum_{i=1}^{N} P_{D G i}=P_{\text {total }} \\
0 \leq P_{D G i} \leq P_{\text {real }-W T, i} \\
0 \leq P_{D G i} \leq P_{\text {real }-P V, i}
\end{array}\right.
$$

Where, $F_{i}$ represents the dispatching cost of distributed new energy supply $i$.

The Lagrangian function corresponding to this optimization problem is as follows:

$$
\begin{gathered}
L=\sum_{i=1}^{N} L_{i}\left(P_{D G i}, \lambda, \mu\right) \\
L_{i}=F_{i}+\lambda_{i}\left(\sum_{i=1}^{N} P_{D G i}-P_{\text {total }}\right)+\mu_{i}\left(P_{D G i}-P_{\text {real }-W T, i}\right) \\
L_{i}=F_{i}+\lambda_{i}\left(\sum_{i=1}^{N} P_{D G i}-P_{\text {total }}\right)+\mu_{i}\left(P_{D G i}-P_{\text {real }-P V, i}\right)
\end{gathered}
$$

Where $L$ represents the Lagrangian global function of the optimization problem, and $L_{i}$ represents the Lagrangian subfunction for the optimization problem of distributed wind power and distributed photovoltaic. $\lambda$, 
$\mu_{i}$ represents the Lagrangian multiplier in the suboptimization problem.

The distributed sub-gradient algorithm is based on information interaction between distributed new energy sources to seek optimal convergence:

$$
\begin{gathered}
P_{D G i}(k+1)=\sum_{j=1}^{N} a_{i j} P_{D G j}(k)-\alpha(k) \times \frac{\partial L_{i}}{\partial P_{D G i}} \\
\lambda_{i}(k+1)=\sum_{j=1}^{N} a_{i j} \lambda_{i}(k)-\alpha(k) \times \frac{\partial L_{i}}{\partial \lambda_{i}} \\
\mu_{i}(k+1)=\sum_{j=1}^{N} a_{i j} \mu_{i}(k)-\alpha(k) \times \frac{\partial L_{i}}{\partial \mu_{i}}
\end{gathered}
$$

Where, $P_{D G j}(k), \lambda_{i}(k), \mu_{i}(k)$ represent the first $\mathrm{k}$ step decision variablei of distributed new energy supply, $a(k)$ represents the iterative step size of step $\mathrm{k}$.

Each distributed new energy supply calculates the sub-gradient direction of its Lagrangian function and receives information from neighboring agents and iterative variables $k$, and then update its decision variables to make the problem reach the global optimal solution.

\section{Example analysis}

\subsection{The example scenario}

In order to verify the effectiveness of the proposed method, this paper uses seven distributed new energy aggregators in the IEEE33 distribution grid for optimal dispatching simulation. Among them, WT is distributed wind power, PV is distributed photovoltaic, and each distribution's operating parameters of the power supply are shown in Table 1. The information transmission link between distributed new energy sources is shown in Figure.2.

Table 1. Parameters of different distributed aggregators

\begin{tabular}{cccccccc}
\hline Parameters & 1 & 2 & 3 & 4 & 5 & 6 & 7 \\
\cline { 1 - 3 }$\alpha$ & 0.02 & 0.03 & 0.04 & 0 & 0.02 & 0.03 & 0.036 \\
$\beta$ & 11 & 8 & 6 & 11.5 & 11.3 & 12 & 9 \\
$\begin{array}{c}\text { Rated } \\
\text { capacity } / \mathrm{kW}\end{array}$ & 200 & 200 & 200 & 200 & 280 & 280 & 280 \\
\hline
\end{tabular}

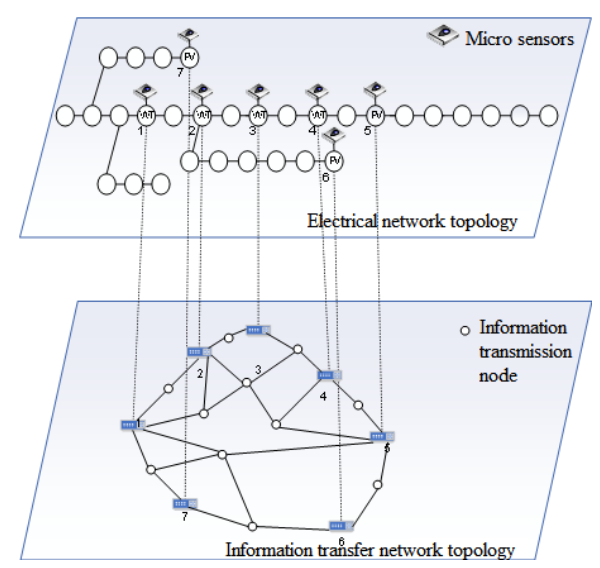

Figure 2. Distribution network topology

In the figure, the two information transmission nodes represent the path length of one unit. In order to ensure the accessibility, timeliness and accuracy of information, and considering the transmission distance limitation of a single transmission link, sometimes two distributed new energy sources need to pass through multiple information transmission nodes to realize information interaction. In this network, the information transmission matrix and its double random matrix are as follows:

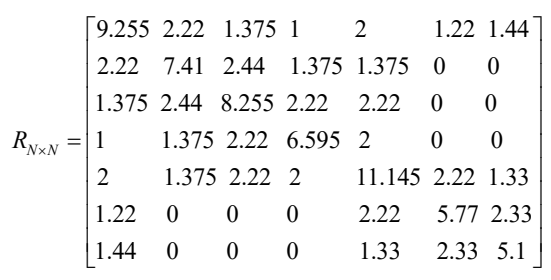

$$
A_{N \times N}=\left[\begin{array}{lllllll}
0.5000 & 0.1199 & 0.0743 & 0.0540 & 0.1080 & 0.0659 & 0.0778 \\
0.1199 & 0.5176 & 0.1704 & 0.0960 & 0.0960 & 0 & 0 \\
0.0743 & 0.1704 & 0.4911 & 0.1321 & 0.1321 & 0 & 0 \\
0.0540 & 0.0960 & 0.1321 & 0.5508 & 0.1670 & 0 & 0 \\
0.1080 & 0.0960 & 0.1321 & 0.1670 & 0.3768 & 0.0751 & 0.0450 \\
0.0659 & 0 & 0 & 0 & 0.0751 & 0.6119 & 0.2471 \\
0.0778 & 0 & 0 & 0 & 0.0450 & 0.2471 & 0.6301
\end{array}\right]
$$

\subsection{Comparative Analysis of Distributed Optimal Dispatch and Centralized Dispatch}

This section verifies the effectiveness of the proposed distributed algorithm by comparing it with the centralized algorithm.

The results of the two algorithms are shown in Table 2

Table 2. Optimal dispatch and centralized dispatch

\begin{tabular}{lcccc}
\hline & $P_{D G 1}$ & $P_{D G 2}$ & $P_{D G 3}$ & $P_{D G 4}$ \\
\cline { 1 - 1 } Distributed dispatch & $44.98 \mathrm{~kW}$ & $84.58 \mathrm{~kW}$ & $102.60 \mathrm{~kW}$ & $56.53 \mathrm{~kW}$ \\
Centralized dispacth & $24.99 \mathrm{~kW}$ & $116.67 \mathrm{~kW}$ & $137.50 \mathrm{~kW}$ & $52.71 \mathrm{~kW}$ \\
& $P_{D G 5}$ & $P_{D G 6}$ & $P_{D G 7}$ & $F$ \\
\cline { 1 - 1 } Distributed dispatch & $39.91 \mathrm{~kW}$ & $21.22 \mathrm{~kW}$ & $66.77 \mathrm{~kW}$ & $4164.1 \$$ \\
\cline { 1 - 1 } Centralized dispacth & $9.99 \mathrm{~kW}$ & $0.00 \mathrm{~kW}$ & $78.13 \mathrm{~kW}$ & $4128.4 \$$ \\
\hline
\end{tabular}


By comparing the results of distributed optimization dispatch and centralized dispatch, we can find that:

(1) The results of power generation planning are slightly different. Compared with centralized dispatch, the distributed optimization dispatching results basically complete the power generation plan. In this mode, each distributed new energy supply can only obtain local information, and iteratively update the decision variables with its own objective function. Therefore, there is a slight error in the calculation result.

(2) In the distributed optimization dispatch, each distributed new energy source takes the lowest power generation cost as the target, so the overall economy is slightly lower than the centralized dispatch, and the relative error is $0.872 \%$. But its open and interconnected environment does not require the construction of a centralized dispatching control center.

\subsection{Analysis of examples under information error and information delay}

Ubiquitous power IoT has the possibility of misinformation and information delay. When there is a random error in the information transmission between the distributed new energy nodes 1 and 2, the data transmitted between the two distributed new energy sources will be deviated and the simulation result is shown in Figure.3. When the information transmission delay between the distributed new energy nodes 1 and 2 is 10 times of the information transmission delay between other nodes, the simulation result is shown in Figure. 4.

Table 3. Outputs of different distributed generations under information error

\begin{tabular}{ccccccc}
\hline$P_{D G 1}$ & $P_{D G 2}$ & $P_{D G 3}$ & $P_{D G 4}$ & $P_{D G 5}$ & $P_{D G 6}$ & $P_{D G 7}$ \\
\hline $0.32 \mathrm{~kW}$ & $1.74 \mathrm{~kW}$ & $125.28 \mathrm{~kW}$ & $90.68 \mathrm{~kW}$ & $65.43 \mathrm{~kW}$ & $42.85 \mathrm{~kW}$ & $89.88 \mathrm{~kW}$ \\
\hline
\end{tabular}

At this time, the total output of distributed new energy source is $416.19 \mathrm{~kW}$. The final total cost is: $\mathrm{F}=$ 4388.3\$.Compared with the case without information error, the economic efficiency of the dispatching result is reduced by $5.67 \%$, but the dispatching mode can still basically complete the overall dispatching task.

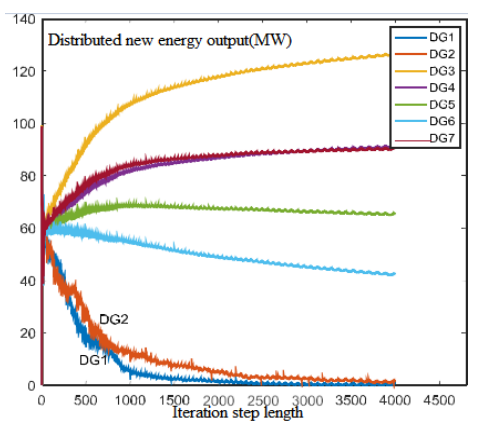

Figure 3. Results under information interrupt

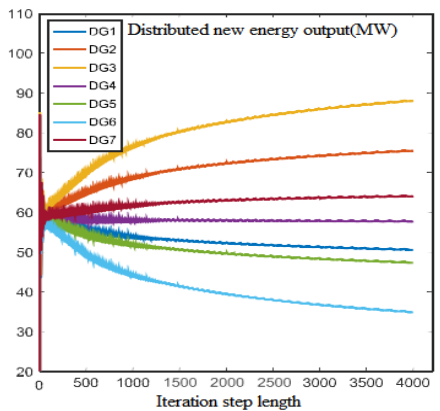

Figure 4. Results under information delay

In the convergence process after considering the information error, due to the error between the distributed new energy sources 1 and 2, the output of the distributed new energy sources 1 and 2 gradually tends to 0 as the algorithm is iterativing. The output values of the distributed new energy sources are shown in Table 3.

Table 4. Outputs of different distributed generations under information delay

\begin{tabular}{ccccccc}
\hline$P_{D G 1}$ & $P_{D G 2}$ & $P_{D G 3}$ & $P_{D G 4}$ & $P_{D G 5}$ & $P_{D G 6}$ & $P_{D G 7}$ \\
\hline $50.68 \mathrm{~kW}$ & $75.05 \mathrm{~kW}$ & $87.41 \mathrm{~kW}$ & $57.69 \mathrm{~kW}$ & $57.51 \mathrm{~kW}$ & $35.32 \mathrm{~kW}$ & $63.88 \mathrm{~kW}$ \\
\hline
\end{tabular}

At this time, the total output of distributed new energy supply is $417.55 \mathrm{~kW}$. The final total cost is: $\mathrm{F}=$ 4255.1\$. This means that in the case of information delay, the dispatching result is $2.18 \%$ lower than that in the case where no information error occurs, but the dispatching mode can also basically complete the total dispatching task.

\section{Example analysis}

In this paper, a distributed optimal dispatching architecture for new energy aggregators is established. In
In the convergence process after considering the information delay, although there is information delay between he distributed new energy sources 1 and 2, as the algorithm is iterating, the whole optimization process still tends to converge, and the output of the new energy supply is shown in Table 4. the power IoT environment, the distributed decentralization concept is used to optimize the dispatching solution, so that distributed iterative convergence is achieved based on information interaction. The results are as follows:

(1) The proposed dispatching architecture and distributed optimization dispatching algorithm have better convergence speed and convergence, and can be applied to the ubiquitous power IoT development interconnection environment.

(2) The algorithm will be affected by information error and information delay during the operation. The two cases will cause the dispatching result of the 
algorithm to deviate from the optimal solution, but it can still guarantee the basic completion of the dispatching task.

\section{Acknowledgments}

This work was financially supported by Smart Grid Protection and Control of NARI Group (Market mechanism of large-scale clean energy consumption across provinces) and State Grid Science and Technology Project (Research on power grid scheduling and transaction model for global energy Internet)

\section{References}

1. XIA Y, LIU J. Review of virtual power plant based on distributed generation $[\mathrm{J}]$. Electric Power Automation Equipment, 2016, 36(4), 100-106.

2. QI S. Accelerate the innovation of safety management mechanism to support the construction of electric power Internet of things [N]. State grid, 2019-09-05(003).

3. SUN H, GUO Q, PAN S. Energy Internet: Concept, Architecture and Frontier Outlook[J] . Automation of Electric Power systems, 2015, 39(19), 1-8

4. Sun Q, Han R, Zhang H, et al. A MultiagentBased Consensus Algorithm for Distributed Coordinated Control of Distributed Generators in the Energy Internet[J]. IEEE Transactions on Smart Grid, 2015, 6(6), 1-1.

5. Hug G, Kar S, Wu C. Consensus + Innovations Approach for Distributed Multiagent Coordination in a Microgrid[J]. IEEE Transactions on Smart Grid, 2015, 6(4), 18931903.

6. SONG S, HUANG $\mathrm{W}$, CHEN $\mathrm{F}$, et al. Design of Distributed Energy Optimization Operation Strategy Based on Cooperative Game [J] . Electrical \& Energy Management Technology, 2015, 35(4), 8-16.

7. JI M, ZHANG P, YAO Y, et al. Distributed Energy Optimization Method for Microgrid Using Market-based Control[J] . Automation of Electric Power systems, 2017, 41(15), 34-41.

8. Yu J, Ni M, Jiao Y, et al. Plug-in and plugout dispatch optimization in microgrid clusters based on flexible communication[J]. Journal of Modern Power Systems and Clean Energy, 2017, 05(04), 663-670.

9. Nedic A, Ozdaglar A. Distributed sub-gradient methods for Multi-Agent optimization[J]. IEEE Transactions on Automatic Control, 2009 , 54(1), 48-61.
10. WANG Ja, LI J, LI D. Distributed subgradient optimization algorithm with communication delays for multi-agent switched networks [J]. Computer science, 2019, 46(7), 81-85 